\title{
Broadband adiabatic light transfer in optically induced waveguide arrays
}

\author{
Charles Ciret, ${ }^{1,2, *}$ Virginie Coda, ${ }^{1,2}$ Andon A. Rangelov, ${ }^{3}$ Dragomir N. Neshev, ${ }^{4}$ and Germano Montemezzani ${ }^{1,2}$ \\ ${ }^{1}$ Université de Lorraine, LMOPS, EA 4423, 2 Rue E. Belin, F-57070 Metz, France \\ ${ }^{2}$ Supélec, LMOPS, EA 4423, 2 Rue E. Belin, F-57070 Metz, France \\ ${ }^{3}$ Department of Physics, Sofia University, James Bourchier 5 Boulevard, 1164 Sofia, Bulgaria \\ ${ }^{4}$ Nonlinear Physics Center, Research School of Physics and Engineering, Australian National University, Canberra ACT 0200, Australia
}

(Received 16 October 2012; published 7 January 2013)

\begin{abstract}
We demonstrate experimentally the broadband and adiabatic light transfer in coupled waveguides based on multiple stimulated Raman adiabatic passage. Our experimental platform utilizes planar-type reconfigurable optically induced waveguide arrays and allows for efficient and robust transfer of light from an input waveguide over multiple states (up to nine) to an output waveguide. The same waveguide structures are tested for two propagation wavelengths separated by more than $200 \mathrm{~nm}$, proving the achromaticity of the transfer process.
\end{abstract}

DOI: 10.1103/PhysRevA.87.013806 PACS number(s): 42.79.Gn, 42.82.Et, 42.50.Xa, 42.65.Hw

\section{INTRODUCTION}

The propagation of light in a series of evanescently coupled optical waveguides has led to many interesting applications in integrated optics [1-4]. As reviewed recently [5], such systems bear utmost fundamental interest due to their important analogies with quantum physics [6]. Among others, several studies have been carried out on optical analogs of Rabi oscillations [7], Landau-Zener tunneling [8-11], and, importantly, stimulated Raman adiabatic passage (STIRAP) [12-15].

The conventional STIRAP process in an atomic $\Lambda$ system involves the transfer of the atomic population from an initial (ground) state, through an intermediate (upper) state, to a final state, where the intermediate state remains entirely unpopulated. This concept can be generalized to a multiple STIRAP process with $N$ number of intermediate states [16-21]. Interestingly, the phenomenon of STIRAP in atomic physics finds a complete mathematical analogy in optics when exploring the propagation-dependent wave coupling in adjacent optical waveguides. This analogy has led to many novel concepts for integrated optics [4,5,15,22-25], which can potentially profit from the fact that the involved adiabatic light transfer provides wideband operation and is insensitive to the exact evolution of coupling coefficients between the waveguides. Adiabatic light transfer has been experimentally demonstrated for a system of three states (one intermediate waveguide) $[14,15]$ and up to five intermediate waveguides [23] using fixed waveguide structures. Even though polychromaticity has been tested for beam splitting applications [4,25], regrettably, all current experiments to test the adiabatic STIRAP-like transfer have only used a single wavelength. Therefore, the biggest advantage, the broadband behavior of the transfer, remains without experimental verification. The test of the adiabaticity of the light transfer process is particularly important in the case of multiple STIRAP when the maximum number of dressed states can be different for the different spectral components. Therefore, it is of great importance to understand the effect of polychromaticity on the process of multiple STIRAP in optical systems.

*charles.ciret@supelec.fr
In this work, we study experimentally the adiabatic and achromatic light transfer between multiple intermediate states in an array of coupled optical waveguides. To test the conditions of adiabaticity for different wavelengths, we develop a new experimental platform that allows for complete flexibility and dynamic reconfiguration of the optical waveguide structure.

Our optical approach is analogous to the generalized multiple STIRAP from quantum physics and hence exhibits high efficiency and robustness. Indeed, we demonstrate adiabatic light transfer over up to 11 waveguides (9 intermediate waveguides) for light propagation in the near infrared. The light transfer process is shown to be highly achromatic, as verified by probing the same structures at wavelengths of 633 and $850 \mathrm{~nm}$. As will be discussed and verified, this is true provided that the number of intermediate states does not exceed the limit for which, depending on the light wavelength, the evolution loses adiabaticity. The experimental findings are shown to be in good agreement with theoretical expectations based on STIRAP theory.

Our experimental platform is based on the optical induction technique in a photorefractive crystal [26], but it employs a side-on illumination technique. This allows us to induce one-dimensional waveguides with arbitrary curvature and geometry in the propagation direction. This is in sharp contrast to the well-known head-on optical induction technique that only allows the formation of stationary [26] or periodically modulated lattices [27,28]. Indeed, the lateral illumination technique has been successfully used for induction of single waveguides [29,30] or sharp lattice boundaries [31]. Here, we used this optical induction to create adiabatically varying coupling between multiple coupled waveguides and to demonstrate adiabatic light transfer at two vastly different wavelengths separated by more than $200 \mathrm{~nm}$.

\section{THEORETICAL BACKGROUND}

The optical system under investigation is shown in Fig. 1 and consists of a planar array of closely spaced waveguides. Initially, light is injected into the optical input waveguide $I$, which is weakly bent to produce a coupling constant toward the neighboring array. In this way, the coupling constant depends on the longitudinal coordinate $z$. The array is composed of 
(b)
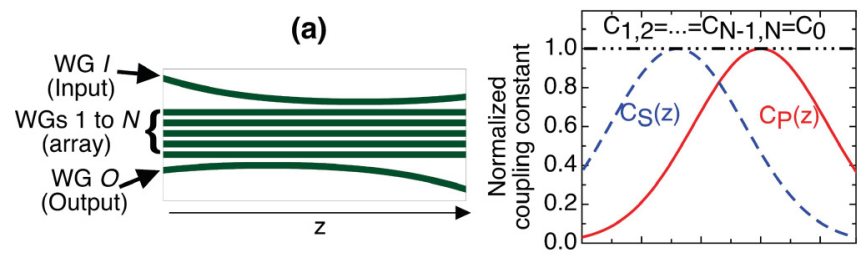

(d)
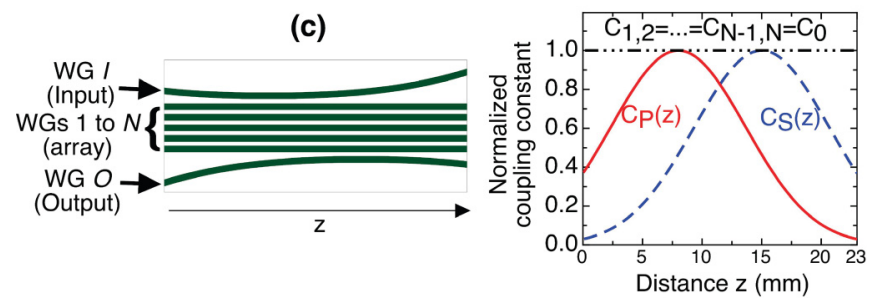

FIG. 1. (Color online) (a) Waveguide structure providing an optical analogy to the multiple STIRAP process, (b) corresponding longitudinal dependence of the normalized coupling constants for the counterintuitive case, and (c,d) corresponding intuitive case. $C_{P}(z)$ is the coupling constant between the input waveguide $I$ and waveguide 1 , and $C_{S}(z)$ is the coupling constant between waveguide $N$ and the output waveguide $O$.

$N$ identical straight waveguides. The output waveguide $O$ is coupled to the last waveguide of the array and is also weakly bent. In paraxial approximation, the propagation of a monochromatic light beam in this kind of structure can be analyzed in the framework of the couple mode theory (CMT), which treats the problem in a discrete way by involving the evanescent coupling between nearest neighbors.

The corresponding evolution of the wave amplitudes can be described by a set of $N+2$ coupled differential equations (in matrix form) $[12,14]$ :

$$
i \frac{d \mathbf{A}(z)}{d z}=\mathbf{H}(z) \mathbf{A}(z),
$$

where $\mathbf{A}=\left(A_{I}(z), A_{1}(z), \ldots, A_{N}, A_{O}(z)\right)^{T}$ is the amplitude of modes in the individual waveguides and

$$
\mathbf{H}(z)=\left(\begin{array}{cccccc}
0 & C_{P}(z) & 0 & 0 & \ldots & 0 \\
C_{P}(z) & 0 & C_{1,2} & 0 & \cdots & 0 \\
0 & C_{1,2} & 0 & \ddots & & \vdots \\
0 & 0 & \ddots & \ddots & C_{N-1, N} & 0 \\
\vdots & \vdots & & C_{N-1, N} & 0 & C_{S}(z) \\
0 & 0 & \cdots & 0 & C_{S}(z) & 0
\end{array}\right)
$$

contains the coupling constants. All the diagonal elements are zero, because we consider waveguides with the same propagation constant (effective refractive index) and the bending of the waveguides $I$ and $O$ is moderate. The geometry of the structure ensures a $z$ dependence of the coupling constants $C_{P}(z)$ (between the input waveguide $I$ and waveguide 1) and $C_{S}(z)$ (between waveguide $N$ and the output waveguide $O)$. Moreover, due to their spatial evolution [represented in Figs. 1(b) and 1(d)], $C_{P}(z)$ and $C_{S}(z)$ are equivalent to the pump and Stokes pulses used in the STIRAP process in quantum physics $[18,20,32]$. In our case, the array of $N$ intermediate waveguides is chosen to be uniform, which leads to $C_{1,2}=\cdots=C_{N-1, N}=C_{0}$. If we map the longitudinal $z$ dependence into time dependence, Eq. (2) is then identical to the time-dependent Schrödinger equation for a chain linkage pattern that generalizes the three state lambda system [18].

The coupling constants are dependent on the physical parameters of the waveguide array and especially on the waveguide spacing. For our specific cases, the relation between the waveguide design and the shape of the $z$-dependent coupling constants in Fig. 1 has been established using the Floquet-Bloch waves formalism [1]. As explained in Sec. III, the peak values of the coupling constant (the smallest distance between the waveguides) and of the coupling constants for other specific waveguide distances have been verified experimentally by studying the discrete diffraction pattern $[3,33]$.

For an odd number $N$ of waveguides in the array, the matrix $\mathbf{H}(z)$ has one eigenvalue that is equal to zero. The eigenvector corresponding to the zero eigenvalue is the so-called dark state or adiabatic transfer state [18-20]. The adiabatic transfer state $a(z)$ is given by

$$
a(z)=\frac{1}{\mathcal{N}}\left(A_{I}(z), 0, A_{2}(z), \ldots A_{N-1}(z), 0, A_{O}(z)\right)^{T},
$$

with

$$
A_{2 k}(z)=\frac{(-1)^{k}}{\mathcal{N}} C_{P}(z) \ldots C_{2 k-2,2 k-1} C_{2 k+1,2 k+2} \ldots C_{S}(z),
$$

where $\mathcal{N}$ is a normalization factor, $A_{I} \equiv A_{0}$, and $A_{O} \equiv$ $A_{N+1}$. In this case, if $a(0)$ is initially excited, then the light will remain within the dark state $a(z)$ throughout the adiabatic evolution $[19,20]$. We can deduce from Eq. (4) the amplitude ratio between the output waveguide $O$ and the input waveguide $I$ :

$$
\begin{aligned}
\frac{A_{O}}{A_{I}}(z) & =(-1)^{N-2} \frac{C_{P}(z) C_{2,3} C_{4,5} \ldots C_{N-1, N}}{C_{1,2} C_{3,4} \ldots C_{N-2, N-1} C_{S}(z)} \\
& =(-1)^{N-2} \frac{C_{P}(z) C_{0}^{(N-1) / 2}}{C_{0}^{(N-1) / 2} C_{S}(z)}=(-1)^{N-2} \frac{C_{P}(z)}{C_{S}(z)},
\end{aligned}
$$

as well as $A_{2 k}(z) / A_{I}(0)=(-1)^{k} C_{P}(z) / C_{0}$. If the propagating constants are ordered counterintuitively, $C_{S}(z)$ before $C_{P}(z)$ [Fig. 1(b)], $C_{P}$ largely exceeds $C_{S}$ at the end of the structure and Eq. (5) shows that no light remains in waveguide $I$. Hence, under such conditions in the adiabatic regime the light will be transferred adiabatically from waveguide $I$ to waveguide $O$ and almost no light will illuminate the remaining waveguides.

The condition for adiabatic evolution in the STIRAP process is given by [18]

$$
\left|\lambda_{p}-\lambda_{q}\right| \gg\left|a^{(p)} \cdot \dot{a}^{(q)}\right| \propto \frac{1}{Z},
$$

where $\lambda_{k}$ are the eigenvalues of $\mathbf{H}(z)$ associated to different adiabatic states and $a^{(k)}$ are the corresponding $z$-dependent eigenvectors, while $Z$ is the spatial overlap of $C_{P}(z)$ and $C_{S}(z)$. The derivative $\dot{a}^{(q)}$ of the $q$ th eigenvector is with respect to the longitudinal spatial coordinate. In the case of an adiabatic transfer state, we have to compare $\lambda_{p}=0$ with the next closest eigenvalue. 


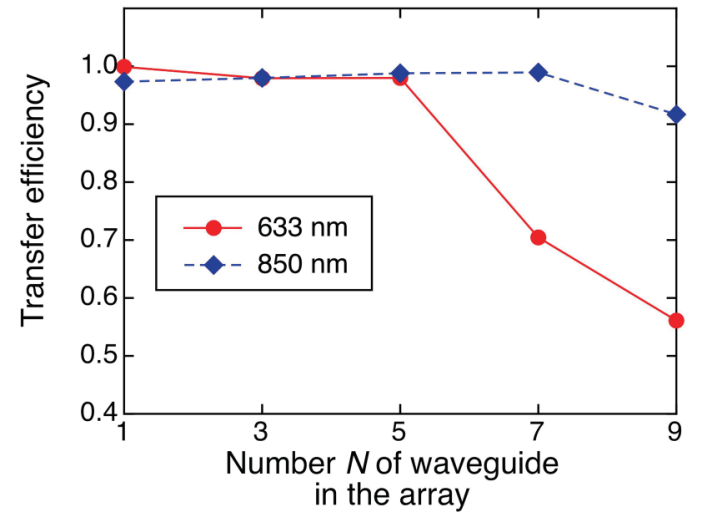

FIG. 2. (Color online) Calculated intensity transfer ratio $I_{O}(z=$ $L) / I_{I}(z=0)$ at the output of the structure represented in Fig. 1(a) as a function of the number of waveguides in the array for two different wavelengths (633 and $850 \mathrm{~nm}$ ).

Due to the shape of the coupling constants [Fig. 1(b)], the latter verify $C_{0} \gg C_{P}, C_{S}$ for most of the propagation, then the two smallest nonzero eigenvalues are approximately given by [18]

$$
\lambda_{ \pm} \approx \pm \sqrt{\frac{C_{\mathrm{P} \max }^{2}+C_{\mathrm{S} \max }^{2}}{n}},
$$

where $n$ is related to the number $N$ of waveguides in the array by $N=2 n-1$ and $C_{\mathrm{P} \max }, C_{\mathrm{Smax}}$ are the maximal values of $C_{P}, C_{S}$. Equation (7) predicts that $\left|\lambda_{ \pm}\right|$decrease as the number of intermediate states increases, i.e., for a larger number of waveguides in the array. Therefore, if $N$ is increased the smallest nonzero eigenvalue gets closer to zero and the adiabatic condition given by Eq. (6) breaks down. In this way, we can define an adiabatic criterion by

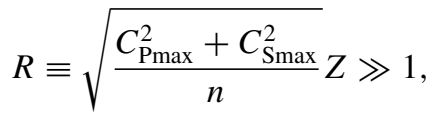

which must be fulfilled in order to have pure adiabatic transfer. Therefore, adiabatic transfer may be expected only up to a finite number $N$ of intermediate waveguides. Beyond this number $\left(N_{\max }\right)$, the transfer loses adiabaticity. Due to the wavelength dependence of the coupling constants, the number $N_{\max }$ depends also on the propagation wavelength. This limiting number is expected to be smaller for shorter wavelengths due to a weaker evanescent coupling (more tightly confined modes). Therefore, if $N$ is not too large, the transfer is expected to be achromatic for a large range of wavelengths.

The above argumentations are illustrated in Figs. 2 and 3, which present calculations under the realistic conditions of our experiments $\left(C_{\mathrm{P} \max }=C_{\mathrm{S} \max }=C_{0}=1.1 \mathrm{~mm}^{-1}\right.$ at $633 \mathrm{~nm}$, $C_{\mathrm{Pmax}}=C_{\mathrm{Smax}}=C_{0}=1.5 \mathrm{~mm}^{-1}$ at $850 \mathrm{~nm}$; see Sec. III). Figure 2 depicts the transfer efficiency as a function of the number of waveguides, $N$, in the array for two different propagating wavelengths $(633$ and $850 \mathrm{~nm})$. The transfer efficiency is defined as the intensity ratio $I_{O}(z=L) / I_{I}(z=0)$ between the output and input waveguides. The efficiency is expected to be close to 1 for both wavelengths up to $N=5$. Then, as expected, it drops faster for the shorter wavelength. Indeed, for $N=7$ the transfer at $633 \mathrm{~nm}$ falls

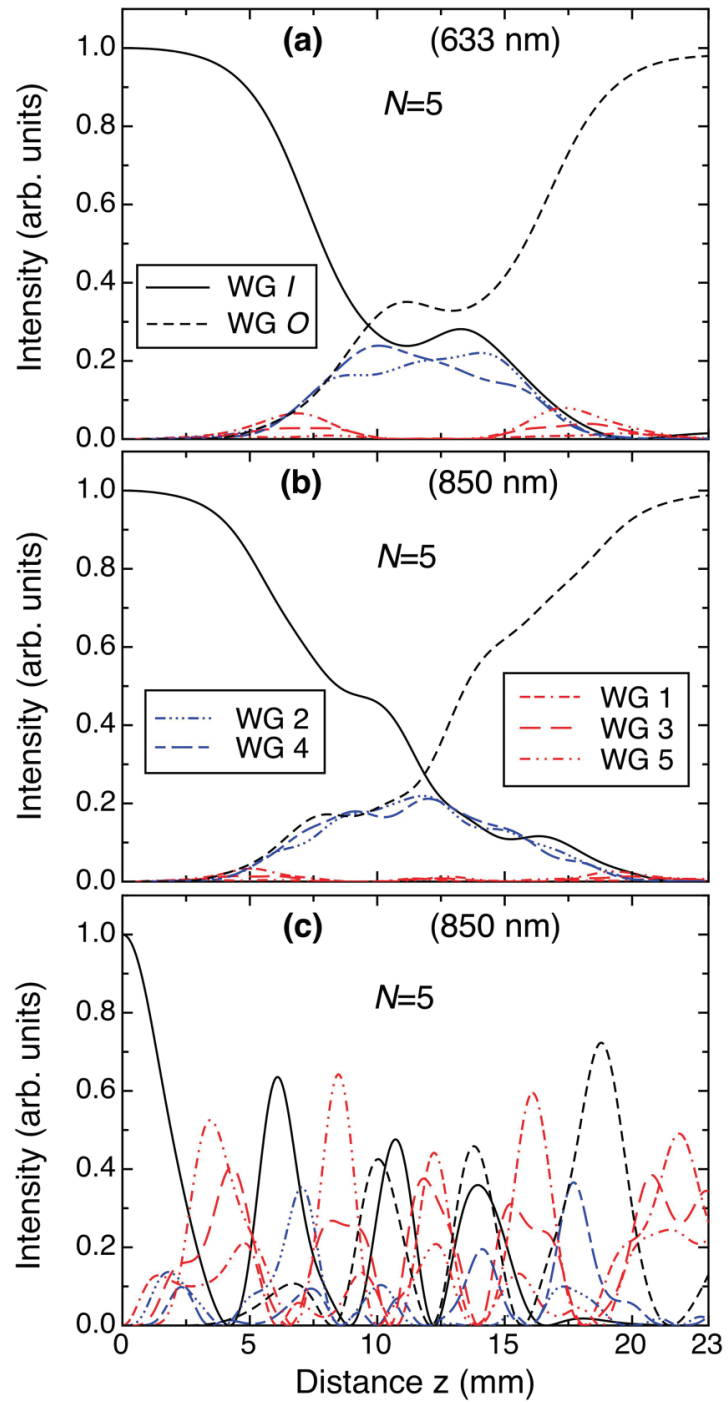

FIG. 3. (Color online) (a,b) Calculated light intensity along the propagation for $N=5$ in the counterintuitive case for light of 633 and $850 \mathrm{~nm}$, respectively. (c) Light evolution in the corresponding intuitive ordering $(N=5)$ at $850 \mathrm{~nm}$.

sharply, while the transfer efficiency remains close to 1 for the 850-nm light. For $N=9$, the efficiency drops also for $850 \mathrm{~nm}$. The corresponding calculated light evolution in the different waveguides is shown in Fig. 3(a) for $N=5$ at $633 \mathrm{~nm}$ and in Fig. 3(b) at $850 \mathrm{~nm}$. One can see that the intermediate states contain almost no light for the odd waveguides, whereas the intensity in the even waveguides increases until half-propagation length and then goes back to zero at the end of the propagation. Figures 3(a) and 3(b) show essentially the same behavior at the wavelengths of 633 and $850 \mathrm{~nm}$, meaning that the optical component is expected to be free from chromatic dependence (achromatic) in the case $N=5$.

Note that, if the coupling constants are arranged in an intuitive [Figs. 1(c) and 1(d)] rather than counterintuitive order, according to theory [18-20] the STIRAP transfer does not occur and the light evolution is more complex, as depicted in Fig. 3(c). It may also be remarked that, if the total number of waveguides is even rather than odd, the matrix $\mathbf{H}(z)$ has no 
zero eigenvalue, and thus a dark adiabatic transfer state does not exist.

\section{EXPERIMENTS}

Experimental verification of the theory for the multiple STIRAP transfer is performed by optically inducing waveguide structures of Figs. 1(a) and 1(c) in a dynamic and reconfigurable way by using a lateral illumination technique [29,30]. The simplified setup of our experiments is shown in Fig. 4. This technique has successfully been used for photoinducing waveguides confined in one or two dimensions, but other photonic structures can be easily realized by changing the intensity pattern on a control light beam.

In our experiments, the image of the desired optical structure is sent to a liquid-crystal spatial light modulator (SLM) working in reflection. The local reorientation of the nematic liquid-crystal molecules rotates the polarization of the 532-nm control illumination according to the picture displayed on the SLM. This polarization pattern is converted into an intensity pattern by means of the polarizing beam splitter and sent toward the sample where the waveguide structure is being photoinduced. The imaging between the SLM and the sample is realized by two crossed cylindrical lenses. The sample used for our experiments is a 23 -mm-long weakly Ce doped $\mathrm{Sr}_{0.61} \mathrm{Ba}_{0.39} \mathrm{Nb}_{2} \mathrm{O}_{6}$ photorefractive crystal (SBN). The photorefractive process leads to a redistribution of charges in

(a)

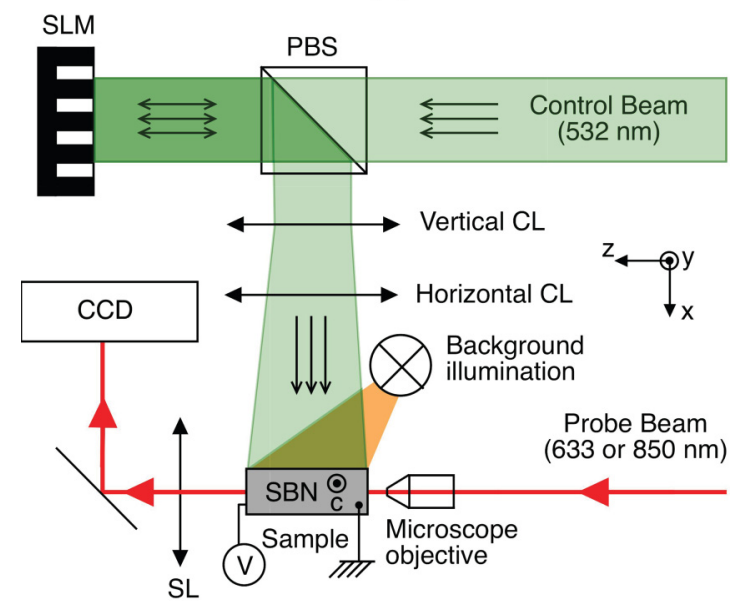

(b)

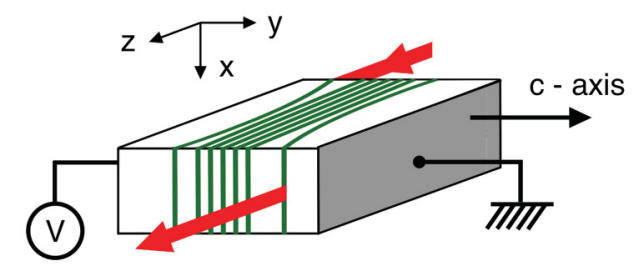

FIG. 4. (Color online) (a) Setup for optical induction of the desired waveguide structures in a photorefractive crystal by a lateral illumination technique. SLM, spatial light modulator; PBS, polarizing beam splitter; CL, cylindrical lens; SL, spherical lens. (b) Close-up of the sample excitation. the crystal and takes place in our case under a bias electric field $E_{0}$ applied parallel to the $c$ axis [Fig. 4(b)].

The formation of a space-charge electric field in response to a localized illumination of a photorefractive material is a complex process that can be successfully modeled for most materials by the Kukhtarev-Vinetskii theory [34]. In our SBN sample, we can safely neglect the charge transport due to the bulk photogalvanic effect. Furthermore, for waveguide widths exceeding 2-3 $\mu \mathrm{m}$, the contribution of carrier diffusion to the charge transport is negligible with respect to the contribution of carrier drift in the applied electric field. In this regime, the equations describing the resulting spatial distribution of the electric field can be exactly solved (for details, see Ref. [35]), and one obtains the simple expression

$$
E_{y}(y, z)=E_{0} \frac{I_{D}}{I(y, z)+I_{D}} .
$$

Here, $I(y, z)$ is the intensity distribution of the control wave coming from the SLM and falling onto the crystal surface. The quantity $I_{D}$ is the so-called "dark intensity," which is associated to the spatially homogeneous component of the conductivity of the crystal. In our specific case, the dark intensity has two contributions. The first is due to the crystal dark thermal conductivity; the second is associated to the homogeneous photoconductivity due to an additional background illumination, which is provided by a halogen white lamp. The use of this background illumination permits a better definition of the waveguide borders [29], as well as the ability to erase faster the old structure before recording a new one. The electric-field distribution of Eq. (9) translates into a change of the refractive index by the linear electro-optic effect, $\Delta n(y, z)=-\left(n^{3} / 2\right) r_{333} E_{y}(y, z)$, where $n$ is the unperturbed refractive index and $r_{333}$ is the electro-optic coefficient active in our experimental configuration. Finally, the refractive index contrast between the zone illuminated by the control wave and the surroundings is $\delta n=\Delta n(I(y, z) \neq 0)-\Delta n(I(y, z)=0)$, that is,

$$
\delta n(y, z)=\Delta n_{0} \frac{I(y, z)}{I(y, z)+I_{D}},
$$

where $\Delta n_{0} \equiv\left(n^{3} / 2\right) r_{333} E_{0}$. The above expression shows that in the zones illuminated by the control wave $I(y, z)$ one obtains a local positive index contrast permitting one to guide a probe wave. The obtained photoinduced waveguides are of the planar type and are confined in the direction of the crystal $c$ axis. Easy reconfiguration of the waveguide structure, in particular the number of the waveguides, is achieved by modifying the pattern on the SLM. In our experiments, the photoinduced structures are probed by a low-power beam, which can be selected at two different wavelengths $(633$ and $850 \mathrm{~nm})$, allowing one to verify the achromaticity and robustness of the multiple STIRAP transfer. Finally, the probe beam output intensity distribution is observed by imaging the exit face of the crystal on a CCD camera, as seen in Fig. 4(a).

In our experiments, the waveguide width is $7.2 \mu \mathrm{m}$, and the period of the array is $9.6 \mu \mathrm{m}$. The applied electric field is $E_{0}=3 \mathrm{kV} / \mathrm{cm}$, leading to a refractive index contrast of $\Delta n \approx 10^{-4}$. The region of overlap between the spatial pulses of coupling constants $C_{P}(z)$ and $C_{S}(z)$, as defined within STIRAP theory, extends over $Z \approx 8 \mathrm{~mm}$. As discussed in 
$[36,37]$, we consider an extension to the coupled mode theory for the moderate coupling regime by introducing effective coupling coefficients between the different waveguides in the coupled mode equations. The effective coupling constants have been estimated experimentally by analyzing the extent of the discrete diffraction pattern $[3,33]$ obtained in a waveguide array composed of 150 waveguides with the same width, period, and $\Delta n$ as the one used for the multiple STIRAP experiments. The high number of excited waveguides after the 23-mm-long propagation permits one to obtain a good estimation of the effective coupling coefficients. For the maximum values (the nearest distance between the waveguides), we obtain $C_{0}(633 \mathrm{~nm})=1.1 \pm 0.1 \mathrm{~mm}^{-1}$ and $C_{0}(850 \mathrm{~nm})=1.5 \pm 0.1$ $\mathrm{mm}^{-1}$; these values normalize the curves of Figs. 1(b) and 1(d) and correspond also to the maximum values of $C_{P}(z)$ and $C_{S}(z)$.

We have first verified experimentally that in the case in which the total number of waveguides is even we cannot obtain an optimum light transfer toward the output waveguide. This result is in full agreement with the theoretical expectations, because no dark state exists in this case. Next, we test experimentally the more important case of an odd total number of waveguides; the results are summarized in Fig. 5. Figures 5(a)-5(g) are for the counterintuitive case and propagation wavelengths of 850 and $633 \mathrm{~nm}$, while Fig. 5(h) is for the intuitive case at $850 \mathrm{~nm}$. The number of intermediate waveguides in the counterintuitive case increases from $N=5$ [Figs. 5(a) and 5(b)] to $N=7$ [Figs. 5(c) and 5(d)] to $N=9$ [Figs. 5(e) and 5(f)] to, finally, $N=11$ [Fig. 5(g)]. The abscissa shows the position of the different waveguides at the end of the crystal. Note that, if only the input waveguide is optically induced (no intermediate waveguides), all the light remains in the input waveguide and exits the crystal at the corresponding position $(I)$.

One may first compare the results of Fig. 5(a) in counterintuitive order of the coupling constants with Fig. 5(h), which is obtained under the same conditions $(850 \mathrm{~nm})$ but for an intuitive order. As expected [see Fig. 3(c)], in the latter case the output light distribution is more complex and distributed strongly over several waveguides, and the transfer toward the final state (waveguide $O$ ) does not occur. This proves experimentally that the counterintuitive order is necessary for an efficient adiabatic light transfer.

When the number $N$ of intermediate waveguides is increased, the efficiency transfer ratio is expected to drop, starting from a certain value of $N$, due to a loss of the adiabatic condition. The drop occurs for larger $N$ at longer wavelengths because the coupling constants are larger than at shorter wavelengths. In our case, the transfer is adiabatic and essentially fully achromatic up to $N=5$ waveguides in the array [Figs. 5(a) and 5(b)]. If we increase $N$ to 7 or 9 , the transfer efficiency to the last waveguide $O$ falls slightly in the case of the shorter wavelength [633 nm, Figs. 5(d) and 5(f)], in agreement with the expectations of Fig. 2. Note that, in the case $N=7$ and for a wavelength of $633 \mathrm{~nm}$, the adiabatic criterion defined by Eq. (8) gives $R \approx 6$. At the same time, the transfer remains essentially unchanged for the near infrared probe beam at $850 \mathrm{~nm}$ [Figs. 5(c) and 5(e)] even though the model calculations predict a drop of the transfer efficiency of the order of $10 \%$ for $N=9$. However, an important drop of
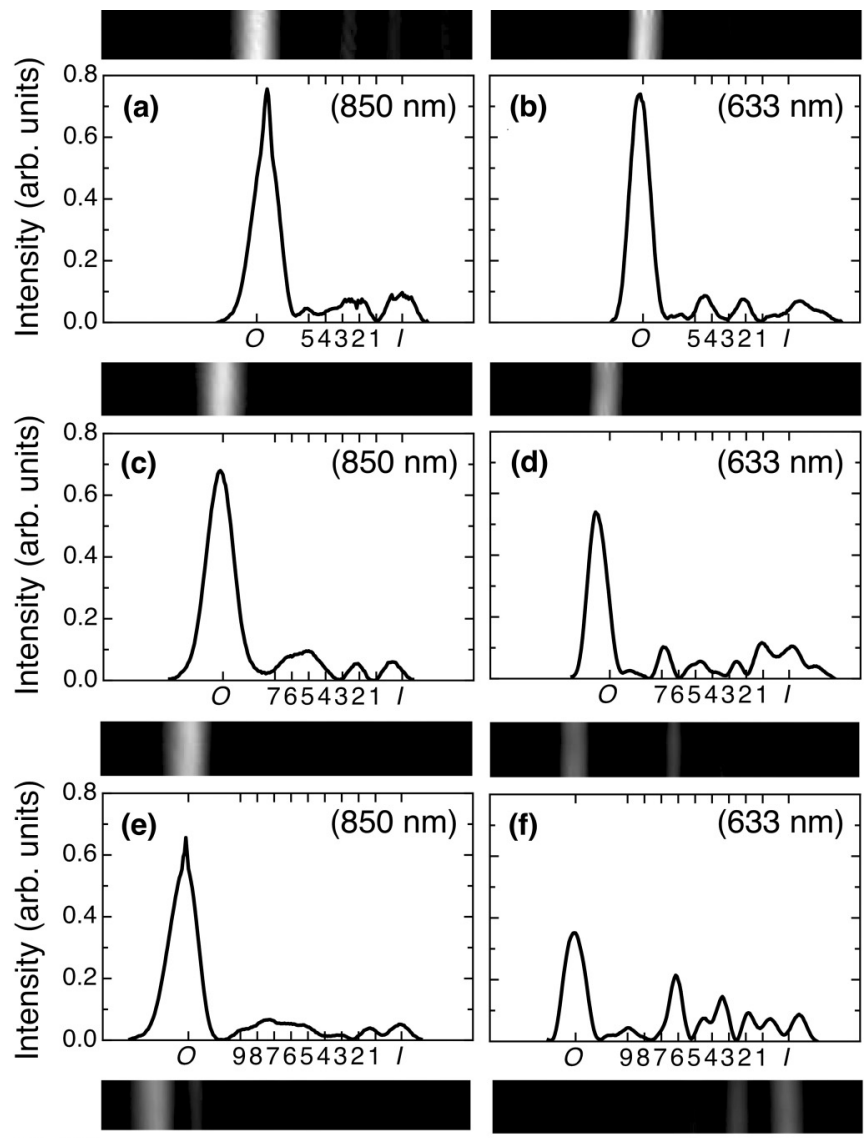

(d)

$(633 \mathrm{~nm})$
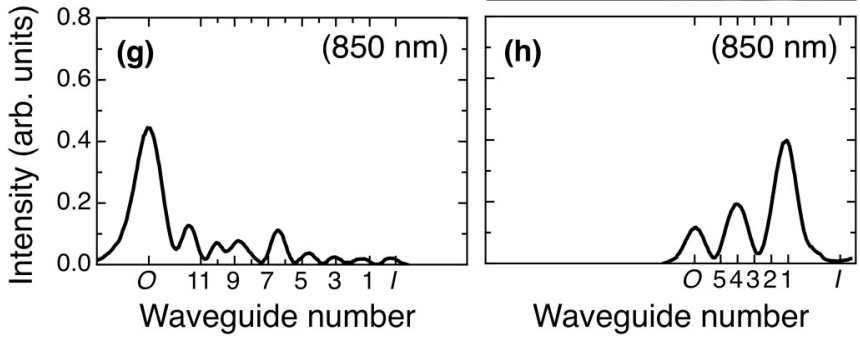

FIG. 5. Experimental output intensity distribution in the counterintuitive case for two different wavelengths [at $850 \mathrm{~nm}$ in (a,c,e,g) and $633 \mathrm{~nm}$ in $(\mathrm{b}, \mathrm{d}, \mathrm{f})]$ : $(\mathrm{a}, \mathrm{b})$ for $N=5,(\mathrm{c}, \mathrm{d})$ for $N=7$, (e,f) for $N=9$, and (g) for $N=11$. (h) Example of intuitive case for $N=5$ at $850 \mathrm{~nm}$.

the transfer efficiency is definitely observed at $850 \mathrm{~nm}$ in the case of $N=11$ [Fig. $5(\mathrm{~g})$ ], for which $R \approx 7$.

Note that, experimentally, the maximum number of intermediate waveguides and the spectral range of achromaticity might be increased further by using longer samples. This would allow one to increase the length $Z$ of the region of spatial overlap between the function $C_{P}(z)$ and $C_{S}(z)$ and thus the ratio between the coupling constants $C_{P}$ and $C_{S}$ and the reciprocal length $1 / Z$, which must be large enough to fulfill the adiabatic condition of Eq. (6).

\section{CONCLUSION}

We have investigated theoretically and experimentally the adiabatic light transfer process taking place over multiple intermediate waveguides in a planar waveguide arrangement, 
which represents a direct analogy with the multiple STIRAP process. In our experiments, we have used photoinduced waveguides recorded in photorefractive materials that permit easy reconfiguration of the structures and a variation of the number of waveguides. This approach is highly versatile and permits study in numerous configurations. It was predicted and confirmed experimentally that the transfer in a given structure is highly achromatic provided that the adiabatic condition is well fulfilled for all the considered probe wavelengths. With increasing number of intermediate states, the adiabatic condition is more difficult to satisfy, especially for the blue side of the spectrum, for which the waveguide modes are more tightly confined and the coupling constants are smaller.

In our specific case, we have shown that the light of wavelength $850 \mathrm{~nm}$ can be successfully transferred to the output waveguide over nine intermediate waveguides, which requires a counterintuitive order for the coupling constants. Up to the case of five intermediate waveguides, the transfer is found to be equally efficient for wavelengths of 633 and $850 \mathrm{~nm}$. The demonstrated broadband adiabatic light transfer opens new possibilities for applications in ultrafast telecommunication devices, where the bandwidth is of particular importance.

\section{ACKNOWLEDGMENTS}

This work has been supported by Australian Research Council and the Bulgarian National Science Fund Grant No. DMU-03/103. C.C., V.C., and G.M. acknowledge financial support from the Contrat de Projets Etat-Région (CPER), project "Matériaux, Energie, Procédés, Produits".
[1] P. Yeh, A. Yariv, and C.-S. Hong, J. Opt. Soc. Am. 67, 423 (1977).

[2] T. Pertsch, T. Zentgraf, U. Peschel, A. Bräuer, and F. Lederer, Phys. Rev. Lett. 88, 093901 (2002).

[3] F. Lederer, G. I. Stegeman, D. N. Christodoulides, G. Assanto, M. Segev, and Y. Silberberg, Phys. Rep. 463, 1 (2008).

[4] F. Dreisow, A. Ornigotti, M. Szameit, M. Heinrich, R. Keil, S. Nolte, A. Tünnermann, and S. Longhi, Appl. Phys. Lett. 95, 261102 (2009).

[5] S. Longhi, Laser and Photon. Rev. 3, 243 (2009).

[6] D. Dragoman and M. Dragoman, Quantum-Classical Analogies (Springer, Berlin, 2004).

[7] S. Longhi, Phys. Rev. A 71, 065801 (2005).

[8] R. Khomeriki and S. Ruffo, Phys. Rev. Lett. 94, 113904 (2005).

[9] S. Longhi, J. Opt. B 7, L9 (2005).

[10] H. Trompeter, W. Krolikowski, D. N. Neshev, A. S. Desyatnikov, A. A. Sukhorukov, Y. S. Kivshar, T. Pertsch, U. Peschel, and F. Lederer, Phys. Rev. Lett. 96, 053903 (2006).

[11] F. Dreisow, A. Szameit, M. Heinrich, S. Nolte, A. Tünnermann, M. Ornigotti, and S. Longhi, Phys. Rev. A 79, 055802 (2009).

[12] E. Paspalakis, Opt. Commun. 258, 30 (2006).

[13] S. Longhi, Phys. Rev. E 73, 026607 (2006).

[14] S. Longhi, G. Della Valle, M. Ornigotti, and P. Laporta, Phys. Rev. B 76, 201101 (2007).

[15] Y. Lahini, F. Pozzi, M. Sorel, R. Morandotti, D. N. Christodoulides, and Y. Silberberg, Phys. Rev. Lett. 101, 193901 (2008).

[16] U. Gaubatz, P. Rudecki, S. Schiemann, and K. Bergmann, J. Chem. Phys. 92, 5363 (1990).

[17] V. S. Malinovsky and D. J. Tannor, Phys. Rev. A 56, 4929 (1997).

[18] N. V. Vitanov, Phys. Rev. A 58, 2295 (1998).

[19] N. V. Vitanov, T. Halfmann, B. W. Shore, and K. Bergmann, Annu. Rev. Phys. Chem. 52, 763 (2001).

[20] N. Vitanov, M. Fleischhauer, B. Shore, and K. Bergmann, Adv. At. Mol. Opt. Phys. 46, 55 (2001).
[21] A. D. Greentree, J. H. Cole, A. R. Hamilton, and L. C. L. Hollenberg, Phys. Rev. B 70, 235317 (2004).

[22] S. Tseng and M. Wu, J. Lightwave Technol. 28, 3529 (2012).

[23] G. D. Valle, M. Ornigotti, T. T. Fernandez, P. Laporta, S. Longhi, A. Coppa, and V. Foglietti, Appl. Phys. Lett. 92, 011106 (2008).

[24] A. A. Rangelov and N. V. Vitanov, Phys. Rev. A 85, 055803 (2012).

[25] C. Ciret, V. Coda, A. A. Rangelov, D. N. Neshev, and G. Montemezzani, Opt. Lett. 37, 3789 (2012).

[26] N. K. Efremidis, S. Sears, D. N. Christodoulides, J. W. Fleischer, and M. Segev, Phys. Rev. E 66, 046602 (2002).

[27] C. R. Rosberg, I. L. Garanovich, A. A. Sukhorukov, D. N. Neshev, W. Krolikowski, and Y. S. Kivshar, Opt. Lett. 31, 1498 (2006).

[28] J. Xavier, S. Vyas, P. Senthilkumaran, C. Denz, and J. Joseph, Opt. Lett. 36, 3512 (2011).

[29] P. Dittrich, G. Montemezzani, P. Bernasconi, and P. Günter, Opt. Lett. 24, 1508 (1999).

[30] M. Gorram, V. Coda, P. Thévenin, and G. Montemezzani, Appl. Phys. B 95, 565 (2009).

[31] N. Malkova, I. Hromada, X. Wang, G. Bryant, and Z. Chen, Opt. Lett. 34, 1633 (2009).

[32] K. Bergmann, H. Theuer, and B. W. Shore, Rev. Mod. Phys. 70, 1003 (1998).

[33] D. N. Christodoulides, F. Lederer, and Y. Silberberg, Nature (London) 242, 817 (2003).

[34] N. V. Kukhtarev, V. B. Markov, S. G. Odulov, M. S. Soskin, and V. L. Vinetskii, Ferroelectrics 22, 949 (1978).

[35] R. Ryf, M. Wiki, G. Montemezzani, P. Günter, and A. Zozulya, Opt. Commun. 159, 339 (1999).

[36] N. Belabas, C. Minot, J. A. Levenson, and J.-M. Moison, J. Lightwave Technol. 29, 3009 (2011).

[37] C. Minot, N. Belabas, J. A. Levenson, and J.-M. Moison, Opt. Express 18, 7157 (2010). 